\title{
Diffraction Properties of Periodic Lattices under Free Electron Laser Radiation
}

\author{
I. Rajkovic, ${ }^{1}$ G. Busse,,${ }^{1}$ J. Hallmann, ${ }^{1}$ R. Moré,${ }^{1}$ M. Petri,,${ }^{1}$ W. Quevedo, ${ }^{1}$ F. Krasniqi,${ }^{2}$ A. Rudenko, ${ }^{2}$ T. Tschentscher, ${ }^{3}$ \\ N. Stojanovic, ${ }^{4}$ S. Düsterer, ${ }^{4}$ R. Treusch, ${ }^{4}$ M. Tolkiehn, ${ }^{5}$ and S. Techert ${ }^{1,2, *}$ \\ ${ }^{1}$ Max Planck Institute for Biophysical Chemistry, 37070 Goettingen, Germany \\ ${ }^{2}$ Max-Planck Advanced Study Group at CFEL, Notkestrasse 85, 22607 Hamburg, Germany \\ ${ }^{3}$ European XFEL GmbH, Albert-Einstein-Ring 19, 22671 Hamburg, Germany \\ ${ }^{4}$ HASYLAB at DESY, Notkestrasse 85, 22607 Hamburg, Germany \\ ${ }^{5}$ Institut fuer Roentgenphysik, Universitaet Goettingen, Friedrich-Hund-Platz 1, 37077 Goettingen, Germany
}

(Received 31 July 2009; published 25 March 2010)

\begin{abstract}
In this Letter, we report the pioneering use of free electron laser radiation for the investigation of periodic crystalline structures. The diffraction properties of silver behenate single nanocrystals $(5.8 \mathrm{~nm}$ periodicity) with the dimensions of $20 \mathrm{~nm} \times 20 \mathrm{~nm} \times 20 \mu \mathrm{m}$ and as powder with grain sizes smaller than $200 \mathrm{~nm}$ were investigated with $8 \mathrm{~nm}$ free electron laser radiation in single-shot modus with $30 \mathrm{fs}$ long free electron laser pulses. This work emphasizes the possibility of using soft x-ray free electron laser radiation for these crystallographic studies on a nanometer scale.
\end{abstract}

DOI: 10.1103/PhysRevLett.104.125503

PACS numbers: 61.80.Cb, 61.05.cp, 64.70.kt

Experiments with soft $\mathrm{x}$-ray radiation $(2-10 \mathrm{~nm})$ are ideal for studying the spatial features in the nanometer scale length. They can be used to determine the electron density distribution of aperiodic systems and to determine the periodic structures of nanoensembles with lattice constants of about 5-10 $\mathrm{nm}$ and bigger, i.e., of periodic selfassembled structures or macromolecular crystals [1,2]. Structural studies on such materials are of particular importance for the development of nanoconfined materials with new and extraordinary physical, chemical, or medical properties [3-5].

The soft x-ray free electron laser FLASH, at the Deutsche Elektronen-Synchrotron (DESY) in Hamburg, is the first extreme ultraviolet to soft $\mathrm{x}$-ray free electron laser (FEL) that provides radiation down to wavelengths as short as $6.5 \mathrm{~nm}[6]$ in the fundamental and is therefore an ideal tool for investigating nanomaterials by means of spectroscopic and diffraction methods [7-11]. Coherent diffraction experiments, such as pioneering imaging experiments of single objects [9], reveal that the outstanding properties of FEL radiation can be used advantageously for studying nanometer-sized structures with soft x-ray radiation.

Here, we report, for the first time, the use of FEL radiation for investigating periodic structures of crystalline nanodimensioned systems. The system investigated was a metallo-organic compound, silver behenate $\left(\mathrm{AgC}_{22} \mathrm{H}_{43} \mathrm{O}_{2}\right)$, expected to show period structures of a length scale fitting the resolution of the experiment in space (5.8 nm lattice dimensions) [12]. The samples were grown as nanocrystals with varying crystal sizes and were investigated as single crystals and powder samples. The periodic structure consists of alternating layers of fatty acid and silver ions with a periodicity of $5.8 \mathrm{~nm}$ (Fig. 1). Silver salts of the long-chain fatty acids are often used in imaging and $\mathrm{x}$-ray diffraction techniques.
The diffraction properties of silver behenate were investigated using $8 \mathrm{~nm}$ free FEL radiation provided at FLASH (30 fs long pulses, in average $4 \times 10^{11}$ photons/pulse). FLASH is operated using the principle of selfamplification of spontaneous emission scheme. The experiments were performed in single-pulse mode. Figure 1 shows a schematic drawing of the soft $\mathrm{x}$-ray diffraction setup used for these experiments. The detailed information about the setup can be found elsewhere [13].

Basic properties of free electron laser diffraction of silver behenate.-In the crystalline phase, silver behenate forms lamellar structures. Figure 1(a) shows a polycrystalline sample. With the experimental configuration sketched in Fig. 1(b), they give rise to a Bragg peak as recorded in Fig. 1(c). The lamellar structure [Fig. 1(d)] is typical for such long carbon chain compounds [12] and is similar to that of fibrils, nanotubes, or liquid crystals [2]. Scanning electron microscopy investigations reveal a needlelike morphology [ $20 \mathrm{~nm}$ in diameter, between 1.5 and $20 \mu \mathrm{m}$ long, Fig. 1(a)]. The size of the powder grown by a different crystallization technique has been determined to be on the order of $100 \mathrm{~nm}$. Figures 1(c) and 2(a) show the (100) Bragg diffraction peak collected in single-pulse mode. It is compared to the diffraction signal from silver behenate powder in Fig. 2(b). For the powder, an accumulation of four to ten shots was necessary to yield an acceptable signal-to-noise ratio. The diffracted intensity of the powder sample is lower in the upper part of the image, most likely due to the combined effect of the horizontal polarization of the incoming FLASH beam (in $y$ direction) and the preferred orientation of the needle-shaped polycrystalline sample as shown in Fig. 1(a). One-dimensional cuts parallel to the scattering angle and through the Bragg diffraction peak and the powder diffraction pattern are shown in Figs. 2(c) and 2(d). The bandwidth of the FLASH pulses is in the range of $0.5 \%$ of the emitted wavelength. There are 


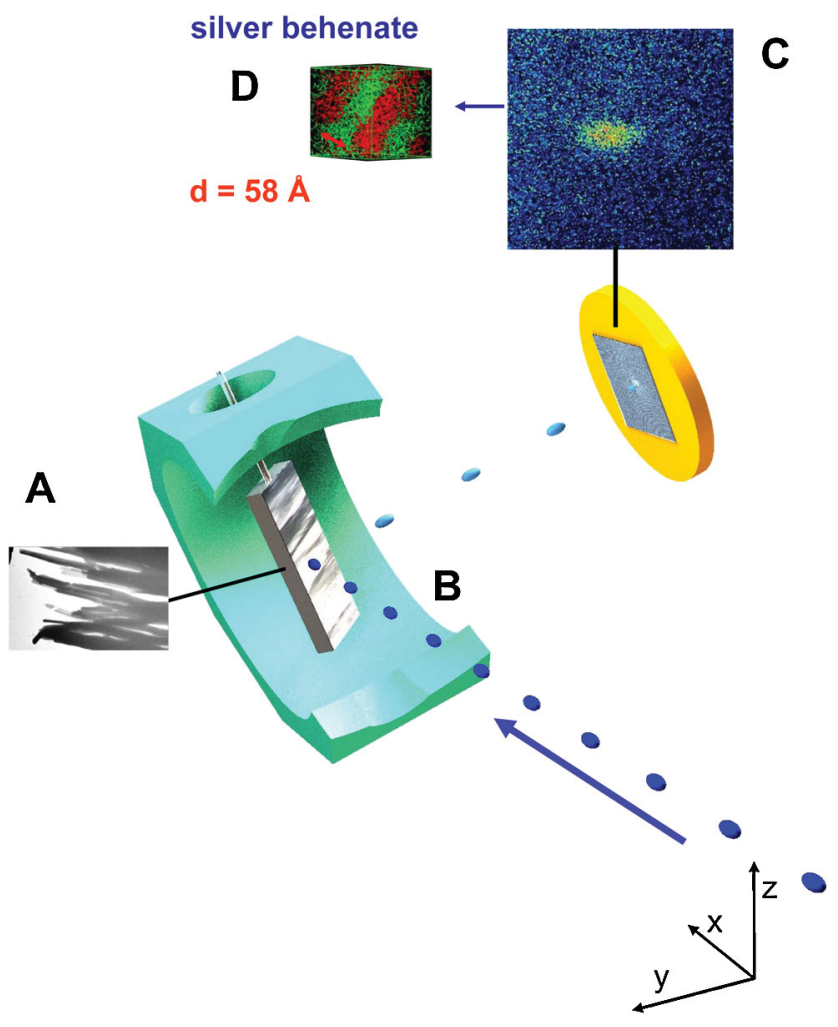

FIG. 1 (color). Experimental setup for the diffraction experiments with FEL radiation. The whole setup is rotated for $20^{\circ}$ around the $x$ axis, and the incoming beam is polarized horizontally in the direction of the $y$ axis. (a) The needlelike morphology of a polycrystalline silver behenate sample has been revealed by scanning electron microscopy. The crystal planes are oriented parallel to the sample surface. (b) FLASH pulses (30 fs, $8 \mathrm{~nm}$, $5 \mathrm{~Hz}$ ) are arriving at the sample at an incident angle of $45^{\circ}$. (c) The $\mathrm{X}$-ray camera is positioned at a $90^{\circ}$ angle to the incident beam, for recording the (100) diffraction peak. (d) Nanoperiodic structure of silver behenate.

several parameters that can affect the monitored diffraction peak width — caused by characteristics of the sample like crystal size and mosaicity, by the FEL source characteristics like bandwidth, divergence, or beam size, and by the function of the apparatus as detector resolution. On the sample side reference measurements reveal no pronounced crystal mosaicity of silver behenate. The effect of crystal size will be discussed in the following. Note that detector resolution, source divergence, and beam sizes do not significantly contribute to the peak broadening. For the experimental configuration used, the source bandwidth translates to instrumental broadening of the Bragg diffraction peak of $1^{\circ}$ (FWHM) on the detector.

Analysis of the structural properties of silver behenate crystallites and powder under free electron laser radiation.-We first analyze the structural properties of silver behenate powder under FLASH radiation by applying Scherrer's formula. This way, it is possible to set the sample size limit which contributes to the diffraction
A Single Crystal
B Powder
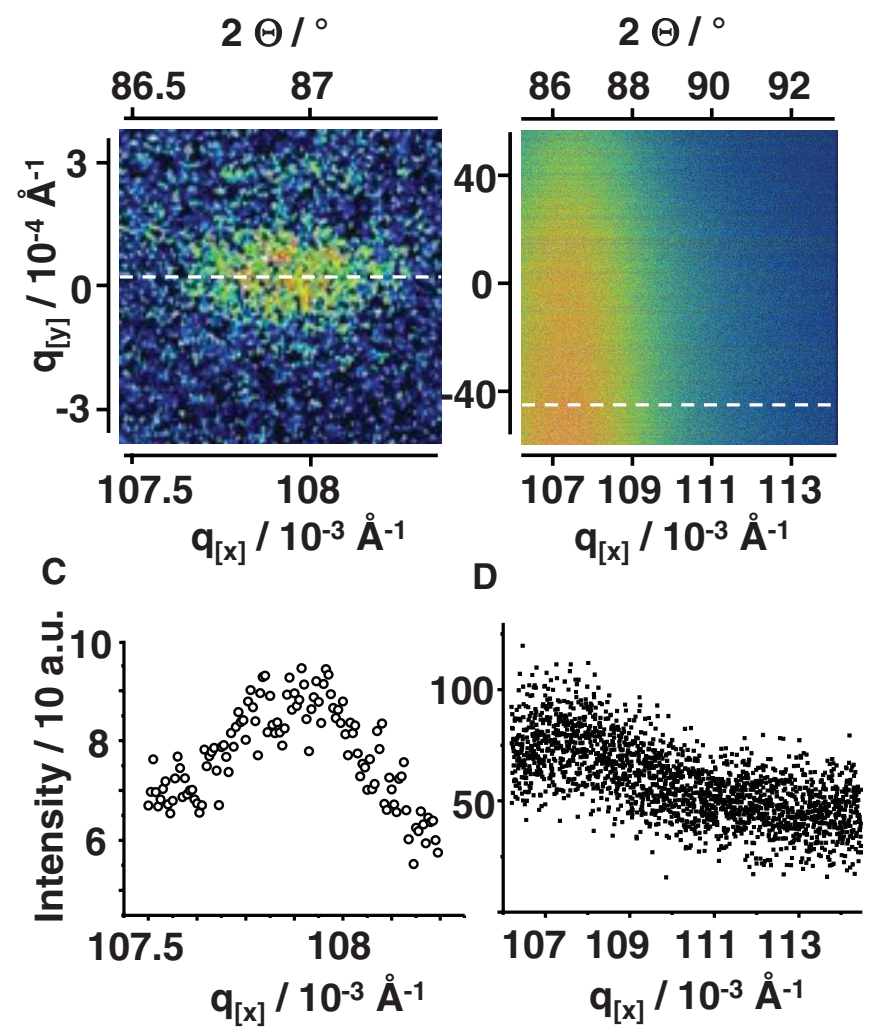

FIG. 2 (color). (a) (100) Bragg diffraction peak of silver behenate investigated by single-shot FEL radiation. (b) Part of the (100) silver behenate powder diffraction ring. Accumulation of 4-10 shots was required to gain a sufficient signal-to-noise ratio. (c) One-dimensional cut through reflection profile [intensity in arbitrary units (a.u.)]. (d) One-dimensional cut through powder diffraction profile. Both cuts were made through the dashed lines shown in (a) and (b).

peak broadening under the experimental conditions used. According to Scherrer's formula, $L=\lambda /\left[\Delta(2 \theta) \cos \left(\theta_{0}\right)\right]$. Here, $L$ is the thickness of the crystallites, $\Delta(2 \theta)$ is the width of the peak (FWHM, rad), $\theta_{0}$ is the Bragg angle of incidence (rad), and $\lambda$ is the FLASH wavelength. From the experimental broadening of the (100) Bragg diffraction peak [Fig. 2(c)] of a single crystal, an average thickness of $L=1.5 \mu \mathrm{m}$ was calculated setting the lower limit of the periodic structure contributing to the Bragg diffraction peak properties.

Contrary to the limit settings discussed for single crystals, the width of the diffraction peak in the powder diffraction pattern [Fig. 2(d)] is more than doubled $\left(\Delta(2 \Theta)=3.5^{\circ}\right)$, which needs to be understood. The powder diffraction pattern is a sum of 4 single-shot images. The pulse-to-pulse wavelength instabilities of the FLASH radiation lead to an accumulated powder diffraction pattern (4 shots) which is artificially broadened. The calculated mean size of the powder grains is $L=200 \mu \mathrm{m}$, which is explained in detail in the supplementary material [14]. 

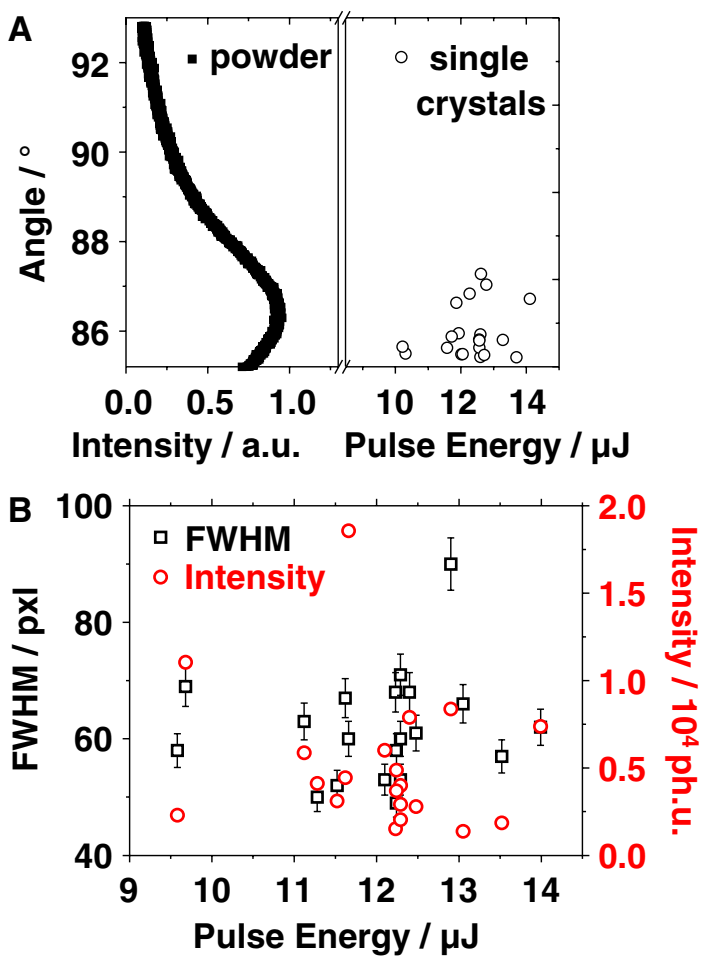

FIG. 3 (color online). (a) Comparison of the radial intensity distribution of the powder diffraction ring (in arbitrary units) with the position of the diffraction spots of various single crystals as a function of FLASH energy. (b) Horizontal FWHM (in number of pixels) and intensities of the Bragg reflections (in photon units) as a function of FLASH energy ( $8 \mathrm{~nm}$ radiation). Note that for the intensity the symbol size has been chosen to reflect the error bar.

Effect of free electron laser peak power on diffraction processes of silver behenate crystallites.-Figure 3 summarizes the properties of the (100) Bragg reflection peak as a function of FLASH energy. As already noted, the position of the (100) Bragg diffraction peaks of silver behenate single crystals fluctuates due to the pulse-to-pulse wavelength fluctuations of FLASH $(8.0 \mathrm{~nm} \pm 0.1 \mathrm{~nm})$ [Fig. 3(a), right-hand side]. Comparison to the powder [Fig. 3(a), left-hand side] gives a coincidence expected due to the FLASH wavelength fluctuations.

Within the resolution of the experiment performed and the x-ray fluxes used $\left(10^{11}\right.$ photons/pulse on a $600 \mu \mathrm{m}$ focal spot), a clear correlation to the pulse power has not been observed. It should be noted that the FLASH power density-though not focused on a 100-200 $\mu \mathrm{m}$ focal spot-was already completely sufficient to remove the metallo-organic sample from the target within $\sim 10$ shots. The presented results yield from true single-shot experiments. Figure 3(b) compares the integral intensity of the (100) Bragg reflection and the FWHM of the reflection for various single crystals as a function of FLASH pulse energy. Also here, the data scatter statistically and no clear correlation has been observed. The symbol size has been chosen to reflect the error bar.

Estimation on the distortion of the crystallites under free electron laser radiation.-One of the major concerns discussed in literature is whether FEL radiation would affect the crystalline properties of matter in such a way that crystallographic studies would not be possible anymore. One should emphasize that the power densities used in these studies were set to $10^{12} \mathrm{~W} / \mathrm{cm}^{2}$ in maximum (in average $2 \times 10^{11} \mathrm{~W} / \mathrm{cm}^{2}$ ), which is in the range or just below the Coulomb explosion limit of $10^{12} \mathrm{~W} / \mathrm{cm}^{2}$ [15]. Nonetheless, the power used was sufficient for single-shot experiments. After damaging, the sample was refreshed. For further discussion it is now assumed that the slowest motions supporting the dissipation of thermal energy and the heating process into the crystal are the excitations of acoustic modes and thermal diffusion processes. With respect to the periodicity of the lattice it is expected that, in particular, the travel of the acoustic waves through the lattice leads to a volume expansion of the lattice which would be observable as a decrease in $d$ spacing. For silver behenate the typical thermal diffusion speed is $v($ diffuse $)=1.67 \times 10^{-3} \mathrm{~m} / \mathrm{s}$ [16]. In the smallest powder grains observable, the diffusion times needed for the thermal energy to travel through the whole grain are then typically $120 \mu \mathrm{s}(L=200 \mathrm{~nm}), 900 \mu \mathrm{s}$ for $1.5 \mu \mathrm{m}$ long crystals, and $12 \mu \mathrm{s}$ for $20 \mathrm{~nm}$ thick single crystals.

Using Young's modulus $E$ and the shear modulus $G$ of waxlike materials (like silver behenate), longitudinal $c_{\text {long }}$ and transversal $c_{\text {trans }}$ speed of sound $c_{\text {long }}=\sqrt{E / \rho}=2 \times$ $10^{5} \mathrm{~cm} / \mathrm{s}$ and $c_{\text {trans }}=\sqrt{G / \rho}=2.5 \times 10^{3} \mathrm{~cm} / \mathrm{s}$ are found [16]. During the 30 fs duration of the FLASH pulse the longitudinal acoustic wave and the transversal acoustic wave therefore can travel only 60 and $0.75 \mathrm{pm}$, respectively, which is on the order of $0.1 \%-1 \%$ of the $d$ spacing. Vice versa, one can estimate how long a longitudinal or transversal mode travels through the investigated powder and crystals. In the powder $(L=200 \mathrm{~nm})$, the longitudinal acoustic wave and the transversal acoustic wave would need $100 \mathrm{ps}$ and $8 \mathrm{~ns}$, respectively. In the crystals, the propagation times are even longer, i.e., 750 ps (longitudinal) and $60 \mathrm{~ns}$ (transversal) for crystals of $1.5 \mu \mathrm{m}$ length and $10 \mathrm{ps}$ (longitudinal) and 800 ps (transversal) for crystals of $20 \mathrm{~nm}$ width. Even if the FLASH pulses produce lattice heating which is dissipated through these acoustic modes and leads to a lattice increase, the duration of the FLASH pulse is too short to observe the listed effects.

Studies with femtosecond optical laser reveal that the lattice of silver behenate can expand up to $10 \%$ within 4 ps. If FEL radiation has a similar effect, there arises the question of how this affects the investigated structure. The described lattice expansion can be monitored as intensity changes of the Bragg reflection, changes in the diffraction angle, and changes in its FWHM. For the diffraction angle changes and the FWHM changes, no clear 
correlation has been found as already stated. In the following, we will discuss the last remaining parameter, i.e., the influence of the FLASH energy on the integral intensity of the Bragg diffraction peaks by performing a onedimensional Fourier analysis [17]. In silver behenate, in one periodic unit the silver carboxylates form a chelate complex (between the colored layers in Fig. 1). They are the dominant scattering centers compared to cigar-shaped electron densities of the carbon chains surrounding. The electron density can be described as a one-dimensional electron density distribution $\rho(u)$ which is defined as $\rho(u)=(1 / a)\left[F_{0}+2 \sum_{h} F_{h} \cos (2 \pi h u / a)\right]$ with $a$ the lattice constant of silver behenate, $u$ the relative coordinate of the silver chelate atoms, $F_{0}$ the predicted structure factor, and $F_{h}$ the experimental structure factor, where $F_{h}^{2}$ is directly proportional to the scattered integral intensity. The simulations based on this Fourier analysis predict an intensity decrease of the (100) Bragg reflection peak by about $25 \%$ if one assumes an increase in $d$ spacing from 5.8 to $6.4 \mathrm{~nm}$ - which corresponds to an $\mathrm{Ag}_{2}-\mathrm{Ag}_{2}$ distance increase of more than $10 \%$. Taking into account the accuracy of integral intensity determination, we have not observed such an effect. Figure 3(b), right-hand side, shows the intensity distribution of the various silver behenate single crystals as a function of FEL energy. The integral intensity varies by a factor of 4 . Taking into account the simulations based on the Fourier analysis, this would correspond to a change in $d$ spacing of about $50 \%$, which is very unlikely. Since no clear correlation of the integral intensity to the FEL energy has been found, the distortion of structures of nanocrystals during the $30 \mathrm{fs}$ long pulses is less than the assumed 15\%-25\% also taking into account that the reflection angles do not change considerably. Therefore, we assign the intensity fluctuations found mainly to the size distribution of the nanocrystals which vary by a factor of 2 . This effect leads to the observed intensity fluctuations by a factor of 4 .

In this work, we have studied for the first time the diffraction behavior of periodic nanoassemblies with FEL radiation. We demonstrated the possibility of using free electron laser radiation for crystallographic studies on the nanometer scale. Under the experimental conditions used, we have shown that the one-dimensional Fourier analysis and Scherrer's equation can still be applied successfully to determine structure properties of single crystals and powders. Analysis of the observed diffraction peak from single crystal samples and from powder samples gave results consistent with the known properties of these samples. Specifically, the broadening of the diffraction peak was consistent with the size of the diffracting crystallites. A calculation of the expected distortion of the crystal on the $30 \mathrm{fs}$ time scale indicated that a negligible distortion should be expected for the FEL pulse energy used. Since no correlation was found between FEL pulse energy and diffraction peak width, the diffraction process was not strongly affected by the FEL peak power.

The studies prove that free electron lasers, similar to state-of-the-art x-ray sources like synchrotrons, bear the potential to be used as highly brilliant $\mathrm{x}$-ray sources for future $\mathrm{x}$-ray science with the advantage of 30 fs short $\mathrm{x}$-ray pulses enabling the real-time monitoring of molecular and chemical structural dynamics.

Special thanks are due to the scientific and technical staff of FLASH at DESY, in particular, to J. Feldhaus, R. L. Johnson, U. Hahn, M. Kuhlmann, M.-T. Nunez, K. Tiedtke, S. Toleikis, E. L. Saldin, E. A. Schneidmiller, and M. V. Yurkov. We also thank the MPG-Advanced Study Group of the CFEL for continuous support and C. Schmidt, A. Hömke, A. Möller, F. Lange, K.-H. Kahlmeyer, B. Wallmann, and R. Bürsing for their technical help with these experiments. This work was supported by DFG/SFB755 Nanoscale Photonic Imaging and EU Grant No. TOTALCRYST/NEST 015592. S. T. is grateful to the Aventis Foundation and the Fonts of the Chemical Industry. W. Q. thanks the DAAD for financial support. F. K., A.R., and S. T. thank the Advanced Study Group of the Max Planck Society for continuous support.

*STECHER@GWDG.DE

[1] A. Guinier, X-Ray Diffraction in Crystals, Imperfect Crystals and Amorphous Bodies (Dover, New York, 1963).

[2] W. Quevedo, M. Petri, G. Busse, and S. Techert, J. Chem. Phys. 129, 024502 (2008).

[3] A. Aharoni, D. Oron, U. Banin, E. Rabani, and J. Jortner, Phys. Rev. Lett. 100, 057404 (2008).

[4] S. Techert, F. Schotte, and M. Wulff, Phys. Rev. Lett. 86, 2030 (2001).

[5] E. Gazit and I. Cherny, Angew. Chem., Int. Ed. 47, 4062 (2008).

[6] W. Ackermann et al., Nat. Photon. 1, 336 (2007).

[7] V. Ayvazyan et al., Eur. Phys. J. D 37, 297 (2006).

[8] E. L. Saldin, E. A. Schneidmiller, and M. Yurkov, The Physics of Free-Electron Lasers (Springer, Berlin, 2000).

[9] H. Chapman et al., Nature Phys. 2, 839 (2006).

[10] S. P. Hau-Riege et al., Phys. Rev. Lett. 98, 145502 (2007).

[11] C. Gahl et al., Nat. Photon. 2, 165 (2008).

[12] K. Binnemans, R. Van Deun, B. Thijs, I. Vanwelkenhuysen, and I. Geuens, Chem. Mater. 16, 2021 (2004).

[13] I. Rajkovic et al., Rev. Sci. Instrum. (to be published).

[14] See supplementary material at http://link.aps.org/ supplemental/10.1103/PhysRevLett.104.125503 for simulation of the powder diffraction broadening as a function of powder grain size

[15] K. Yamanouchi et al., Science 295, 1659 (2002).

[16] http://goldbook.iupac.org/M03966.html.

[17] A. L. Patterson, Phys. Rev. 46, 372 (1934). 